\title{
Searching novel complex solid solution electrocatalysts in unconventional element combinations
}

\author{
Olga A. Krysiak ${ }^{1, \S}$, Simon Schumacher ${ }^{2,3,}$, Alan Savan ${ }^{4}$, Wolfgang Schuhmann ${ }^{1}(\bowtie)$, Alfred Ludwig $\left.{ }^{4,5}(\bowtie)\right)$, and \\ Corina Andronescu ${ }^{2,3}(\triangle)$ \\ ${ }^{1}$ Analytical Chemistry-Center for Electrochemical Sciences (CES), Faculty of Chemistry and Biochemistry, Ruhr University Bochum, \\ Universitätsstr. 150, D-44780 Bochum, Germany \\ ${ }^{2}$ Chemical Technology III, Faculty of Chemistry, Carl-Benz-Straße 199, D-47057 Duisburg, Germany \\ ${ }^{3}$ CENIDE - Center for Nanointegration University Duisburg Essen, Carl-Benz-Straße 199, D-47057 Duisburg, Germany \\ ${ }^{4}$ Chair for Materials Discovery and Interfaces, Institute for Materials, Faculty of Mechanical Engineering, Ruhr University Bochum, Universitätsstr. \\ 150, D-44780 Bochum, Germany \\ ${ }^{5}$ ZGH, Ruhr University Bochum, Universitätsstr. 150, D-44780 Bochum, Germany \\ ${ }^{\S}$ Olga A. Krysiak and Simon Schumacher contributed equally to this work.
}

(c) The Author(s) 2021

Received: 1 February 2021 / Revised: 27 May 2021 / Accepted: 1 June 2021

\section{ABSTRACT}

Despite outstanding accomplishments in catalyst discovery, finding new, more efficient, environmentally neutral, and noble metalfree catalysts remains challenging and unsolved. Recently, complex solid solutions consisting of at least five different elements and often named as high-entropy alloys have emerged as a new class of electrocatalysts for a variety of reactions. The multicomponent combinations of elements facilitate tuning of active sites and catalytic properties. Predicting optimal catalyst composition remains difficult, making testing of a very high number of them indispensable. We present the high-throughput screening of the electrochemical activity of thin film material libraries prepared by combinatorial co-sputtering of metals which are commonly used in catalysis ( $\mathrm{Pd}, \mathrm{Cu}, \mathrm{Ni}$ ) combined with metals which are not commonly used in catalysis ( $\mathrm{Ti}, \mathrm{Hf}, \mathrm{Zr}$ ). Introducing unusual elements in the search space allows discovery of catalytic activity for hitherto unknown compositions. Material libraries with very similar composition spreads can show different activities vs. composition trends for different reactions. In order to address the inherent challenge of the huge combinatorial material space and the inability to predict active electrocatalyst compositions, we developed a high-throughput process based on co-sputtered material libraries, and performed high-throughput characterization using energy dispersive X-ray spectroscopy (EDS), scanning transmission electron microscopy (SEM), X-ray diffraction (XRD) and conductivity measurements followed by electrochemical screening by means of a scanning droplet cell. The results show surprising material compositions with increased activity for the oxygen reduction reaction and the hydrogen evolution reaction. Such data are important input data for future data-driven materials prediction.
\end{abstract}

\section{KEYWORDS}

high-entropy alloys, electrocatalysis, high-throughput screening, thin films, hydrogen evolution reaction, oxygen reduction reaction

\section{Introduction}

For a broad range of different applications, the development of new unexplored materials that show properties like mechanical resistance and durability, high selectivity and stability towards catalytic reactions and efficiency presenting both the performance of the desired properties and acceptable costs is an important mission for creating future technologies. Especially in the energy sector, new and innovative materials that ideally consist of abundant and ecologically favourable elements limiting the use of noble metals are required, for example for efficient electro- and photocatalytic energy conversion or storage.

One material class that attracted considerable attention in recent years due to their advantageous mechanical, physical, and chemical properties are the so-called high entropy alloys (HEAs), also referred to as complex solid solutions (CSSs). HEAs are multielement solid solutions that consist of five or more elements. The combination of more than five different components in an alloy increases the configuration entropy that concomitantly increases the mutual solubility of constituent elements and facilitates formation of solid solutions, sometimes as a single phase. Additionally, the combination of the properties of elements and their interactions leads to final properties of the CCS that are not available for single elements or their simple combinations [1-3]. Various research groups are recently reporting the characterization of specific multielement alloys having unique properties, for example a high catalytic activity or stability $[4,5]$, showing exceptional mechanical strength and ductility [6], or describing novel synthesis methods of multinary alloys [7-10]. As

Address correspondence to Wolfgang Schuhmann, wolfgang.schuhmann@rub.de; Alfred Ludwig, alfred.ludwig@rub.de; Corina Andronescu, corina.andronescu@uni-due.de 
possible catalyst materials, high entropy materials were already successfully applied to the hydrogen evolution reaction (HER) [5, $9,11]$, the oxygen evolution reaction (OER) [11,12], carbon monoxide reduction [13], carbon dioxide reduction $[13,14]$ and the oxygen reduction reaction (ORR) [15-17], methanol oxidation $[18,19]$, ammonia synthesis and decomposition $[20,21]$.

Considering the huge number of different possible combinations of elements and their relative concentrations in a HEA and the variety of the properties those compositions may present, high-throughput screening methods for the analysis of compositional, structural and catalytic effects are indispensable to identify potentially active catalysts for the desired electrocatalytic reactions. The combination of the synthesis of HEA in thin-film material libraries (MLs) consisting of continuous composition spreads where each constituent element ranges from about 10 at.\% to 35 at.\%, with fast electrochemical screening techniques like the scanning droplet cell can lead to a better understanding of how quinary element mixtures interact to exhibit unexpected properties on one hand, and to a quicker identification of suitable electrocatalysts for different reactions on the other [22,23]. In this manner it is also interesting to include elements in unusual combinations that were not tried before because of poor performance of the individual elements, but which might contribute to synergetic effects in CSS and by this to an increased electrocatalytic activity. The selection of an elemental composition as a good starting point for high-throughput screening, without prior knowledge how the interaction in a complex solid solution will later impact on the measured properties, i.e., the catalytic activities, is an unsolved issue. One approach would be to start from the known and assume that the combinations of properties of highly active metals or binary mixtures will be relevant for the activity of complex solid solutions based on 5 or 6 elements. This approach sounds knowledge-driven but fails due to the not known complex interaction of different parameters. The second is to start with a class of metals (e.g., with high activity for some of the reactions) and another class which is typically not found in electrocatalysts but as support materials in heterogeneous catalysis. In this contribution we took the second approach.

We report that the hexanary system (TiNi)-Cu-Hf-Pd-Zr shows composition-dependent activities for the ORR and HER in alkaline media, which were chosen as model reactions due to their importance in the field of energy conversion. The choice of elements was based on the idea to expand the list of elemental compositions which were tested for catalytic activity by combining elements commonly used in catalysis $(\mathrm{Ni}, \mathrm{Cu}, \mathrm{Pd})$ with those which are generally not considered to be promising electrocatalysts (Hf, Zr, Ti).

\section{Experimental}

\subsection{Preparation of thin-film materials libraries}

Thin-film material libraries were prepared by co-sputtering from five confocal sources in a commercial sputter system (CMS 600/400 LIN, DCA Instruments). Polished single-crystal, (100) orientation Si (Siegert Wafer, Germany) with $100 \mathrm{~mm}$ diameter was used as substrates, located at the confocal sputtering position. A $500 \mathrm{~nm}$ thick wet thermal $\mathrm{SiO}_{2}$ layer provided a barrier against potential substrate reactions. The $100 \mathrm{~mm}$ diameter sputter targets were single elements of $\mathrm{Cu}$ (99.99\%, Sindlhauser Materials), Hf (99.95\%, Kaistar R\&D), Pd (99.99\% SenVac), Zr (> 99.2\% Grade 702, K.J. Lesker) and one custom-made $\mathrm{TiNi}_{46.9}$ (FHR). Substrates were loaded through a loadlock to the deposition chamber having a base vacuum of $7 \times 10^{-7} \mathrm{~Pa}$ and $150 \mathrm{~nm}$ of the materials were codeposited at $0.67 \mathrm{~Pa}$ in $\mathrm{Ar}(99.9999 \%)$ at $25^{\circ} \mathrm{C}$ with no further intentional heating. The individual cathode powers were adjusted to produce a centerpoint composition of Ti 14 at.\%, Ni 17 at.\%, $\mathrm{Cu} 16$ at.\%, $\mathrm{Zr} 21$ at.\%, Pd 17 at.\%, Hf 15 at.\% with a total deposition rate of $0.1 \mathrm{~nm} / \mathrm{s}$.

\subsection{Chemical and structural characterization}

Mapping of elemental composition was determined using energydispersive X-ray spectroscopy (EDS, Inca X-act, Oxford Instruments) in a scanning electron microscope (SEM, JSM 5800, JEOL) equipped with an automated stage and operated at $20 \mathrm{keV}$ acceleration voltage. Co was used as a calibration standard, the $\mathrm{Hf}$ $\mathrm{L} \alpha$ peak was used during quantification to avoid confounding with the $\mathrm{Si} \mathrm{K} \alpha$ peak and the composition accuracy was estimated to be about 1 at.\%.

The as-deposited phase constitution was investigated by X-ray diffraction (XRD, D8 Discover, Bruker) using a two-dimensional 2D detector (Vantec-500) in Bragg-Brentano geometry and $\mathrm{Cu}$ $\mathrm{K} \alpha$ radiation. Three frames were acquired with a $2 \theta$ offset of $10^{\circ}$ at discrete angular steps $\left(\theta / 2 \theta+\right.$ offset: $\left.20^{\circ} / 50^{\circ}, 30^{\circ} / 70^{\circ}, 40^{\circ} / 90^{\circ}\right)$ to avoid substrate diffraction peaks.

\subsection{Electrochemical characterization}

MLs were analysed using a high-throughput scanning droplet cell (SDC), which allows site-localized characterization. All electrochemical measurements were conducted in $0.1 \mathrm{M} \mathrm{KOH}$ electrolyte in a three-electrode system containing an $\mathrm{Ag}|\mathrm{AgCl}| 3 \mathrm{M}$ $\mathrm{KCl}$ and $\mathrm{Pt}$ wire as a reference and counter electrode, respectively. For the ORR, linear sweep voltammetry (LSV) was performed between $1 \mathrm{~V}$ and $200 \mathrm{mV}$ vs. reversible hydrogen electrode (RHE) with a scan rate of $10 \mathrm{mV} \cdot \mathrm{s}^{-1}$ and for the hydrogen evolution reaction between $0 \mathrm{~V}$ and $-700 \mathrm{mV}$ vs. RHE. All potentials are reported versus the RHE according to the following equation

$U_{\mathrm{RHE}}(\mathrm{V})=U_{(\mathrm{Ag}|\mathrm{AgCl}| 3 \mathrm{M} \mathrm{KCl})}+0.210+(0.059 \cdot \mathrm{pH})$

where $U_{(\mathrm{Ag}|\mathrm{AgCl}| 3 \mathrm{M} \mathrm{KCl})}$ is the potential measured vs. $\mathrm{Ag}|\mathrm{AgCl}| 3 \mathrm{M}$ $\mathrm{KCl}$ reference electrode, and $0.210 \mathrm{~V}$ is the standard potential of the $\mathrm{Ag}|\mathrm{AgCl}| 3 \mathrm{M} \mathrm{KCl}$ reference electrode at $25^{\circ} \mathrm{C}$. 0.059 is the result of $(R T) \cdot(n F)^{-1}$, where $R$ is the gas constant; $T$ is the temperature $(298 \mathrm{~K}) ; F$ is the Faraday constant and $n$ is the number of electrons transferred during the reaction.

\section{Results and discussion}

Two MLs (ML1 and ML2) with very similar composition spreads were prepared using combinatorial co-sputtering from five different confocal targets. As shown in Fig. S1 in the Electronic Supplementary Material (ESM), the differences between ML1 and ML2 are in the range of 1 at.\% of particular elements. Highthroughput XRD of the as-deposited state revealed no discernable diffraction peaks, with only a broad hump located around $2 \theta$ of approximately $40^{\circ}$, indicative of amorphous materials (Fig. S2 in the ESM). For the electrochemical analysis of the different element compositions a scanning droplet cell in $0.1 \mathrm{M} \mathrm{KOH}$ was used. In this device a $\mathrm{Ag} / \mathrm{AgCl} / 3 \mathrm{M} \mathrm{KCl}$ reference electrode and a $\mathrm{Pt}$ counter electrode are inserted in a poly(methyl methacrylate) (PMMA) conical body that has in addition to a circular tip opening with a diameter of $1 \mathrm{~mm}$, an electrolyte inlet and outlet. The SDC head is mounted on robotic arms and coupled with a force sensor, which allows precise positioning above the ML, then pressing of the head tip to the sample surface, and thus creating a working electrode in each of the measurement areas (MAs). This analytic device is described in more details in Ref. [24]. This highthroughput electrochemical technique allows determination of the activity at 342 discrete MAs of the ML and thus extracts a data map of electrochemical response in correlation with controlled and defined composition trends. Maintaining the same 
measurement conditions and the high data accuracy and reliability due to the automated non-manual operation enables a valid comparison of the activity between the different MAs.

Due to potential surface oxidation of compositions containing $\mathrm{Ti}$, Hf and Zr we adjusted the start potential of the LSV to avoid oxide formation and focused on reduction reactions where any surface oxidized species would be re-reduced before the potential range of the investigated reactions were reached. To confirm the reliability of the measurements, ML2 was measured a second time after a physical $90^{\circ}$ rotation, to rule out any instrument bias effect possibly introduced by the sequential and repeated measurements. Indeed, all activity trends are consistent (Fig. S3 in the ESM) and can be reliably related to the changes of the elemental concentrations. Figure 1 presents electrocatalytic activity maps, obtained by plotting the measured current values at a certain potential with respect to the location on the ML. For ORR a potential of $570 \mathrm{mV}$ vs. RHE, and for HER a potential of -700 $\mathrm{mV}$ vs. RHE were chosen. This allows us to observe how the activity is changing through the whole studied composition space, showing both general trends and location of the high activity areas. Activity for ORR increases from the upper end of the ML1 to the lower end with three highly active regions in the centre-left side of the ML. For HER the general trend shows increase in activity on the diagonal from the upper right side to the lower right side and the high activity area is located in the upper left side of the ML2. The lower panel of Fig. 1 demonstrates the measured LSVs for the ORR and HER at selected MAs from regions with different activities (marked in Fig. 1 with squares).

For the ORR the highest activity was obtained in a small area clustered in the centre of ML1, which corresponds to the composition $\mathrm{Ti}_{14} \mathrm{Ni}_{17} \mathrm{Cu}_{16} \mathrm{Zr}_{21} \mathrm{Pd}_{17} \mathrm{Hf}_{15}$. Moreover, the activity seems to be boosted by the increased amounts of $\mathrm{Ti}$ and $\mathrm{Ni}$ and slightly enhanced with an increased amount of $\mathrm{Cu}$ (see Fig. S1 in the ESM). It is also important to note here, that the LSV curves recorded for the ORR exhibit characteristics of a HEA multi-wave shape as described theoretically [25] and confirmed experimentally [26]. The random arrangement of elements in a CSS results in a continuous and unique adsorption energy distribution pattern. When only on-top adsorption of reaction intermediates is considered, there are the same number of adsorption peaks as the number of elements, with each of those peaks formed by adsorption energies of active sites with one specific element in the centre. On the other hand, considering active sites formed by two or three atoms, the number of adsorption peaks increases as the number of possible centres increases. Each of those multiple adsorption peaks will lead to one current curve, activity of which depends on the position of the peak maximum in relation to the optimal binding energy. The intensity of current curves is defined by the molar ratio of elements and activity driven by the difference of the corresponding peak position from optimal binding energy. When the differences between particular adsorption energy peaks are big enough, an electrochemical response wave can be observed for each adsorption peak. It is important to note that some of those waves might not be visible in the LSV, due to their low activity and high overpotentials. Following these considerations, additional information about the catalytic activity can be acquired by plotting the activity maps at different potentials.

As can be seen in the top panel of Fig. 2, plotting the activity map of ML1 at $820 \mathrm{mV}$ reveals the opposite trend at $570 \mathrm{mV}$. At higher potentials the activity increases with increased amounts of $\mathrm{Ni}$ and $\mathrm{Ti}$, while for lower potentials the activity increases when the amounts of $\mathrm{Ni}$ and $\mathrm{Ti}$ decrease and the amount of $\mathrm{Pd}$ increases. Additionally, the small high activity region, close to the centre of ML, is no longer visible at $570 \mathrm{mV}$ vs. RHE. These results may be explained by the multi-wave shape of the LSV plots. The potential of $820 \mathrm{mV}$ vs. RHE is located in or close to the catalytic region of the first wave, while the potential of $570 \mathrm{mV}$ vs. RHE is instead in the second wave, indicating that for the occurrence of the first wave adsorption sites containing $\mathrm{Cu}, \mathrm{Ni}$ and $\mathrm{Ti}$ is responsible, while the second wave corresponds to the adsorption sites containing mostly $\mathrm{Pd}$, $\mathrm{Hf}$ and $\mathrm{Zr}$.

\section{(a)}
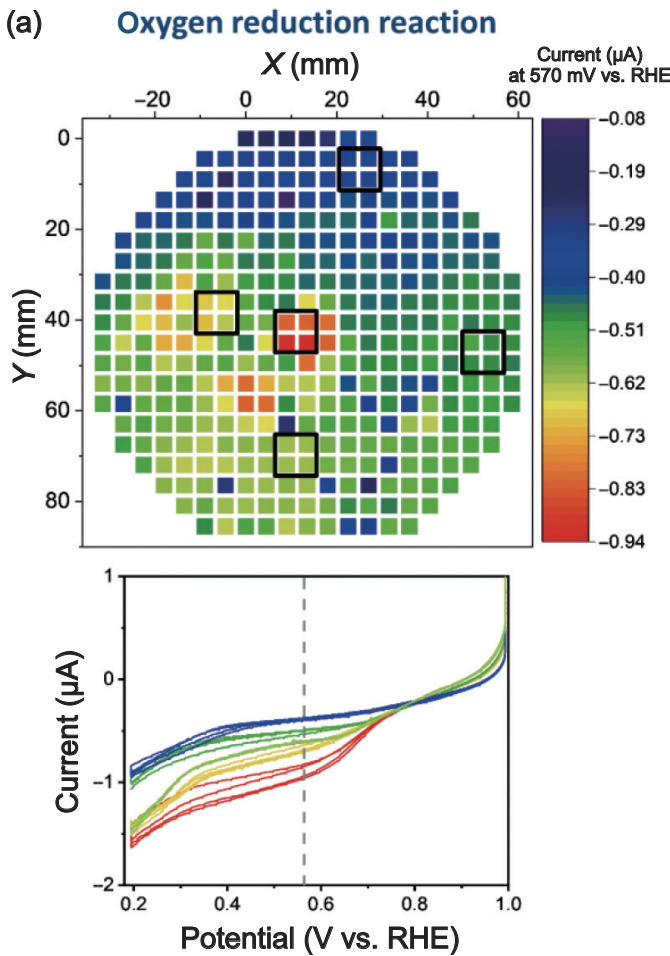

(b) Hydrogen evolution reaction

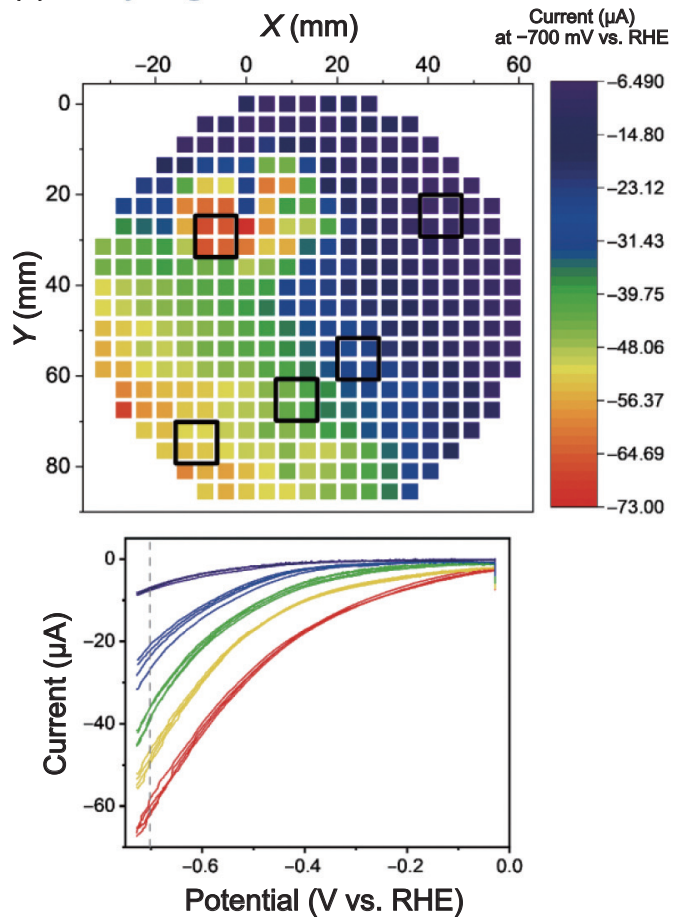

Figure 1 Comparison of activity maps obtained for the ORR (a) and the HER (b). Individual LSVs of the selected MAs characterized by different electrochemical activities (indicated by squares) are presented on the bottom part of the figure. For the ORR the current at $570 \mathrm{mV}$ vs. RHE and for the HER the current at $-700 \mathrm{mV}$ vs. RHE (dashed lines) were selected as the measure of activity. 

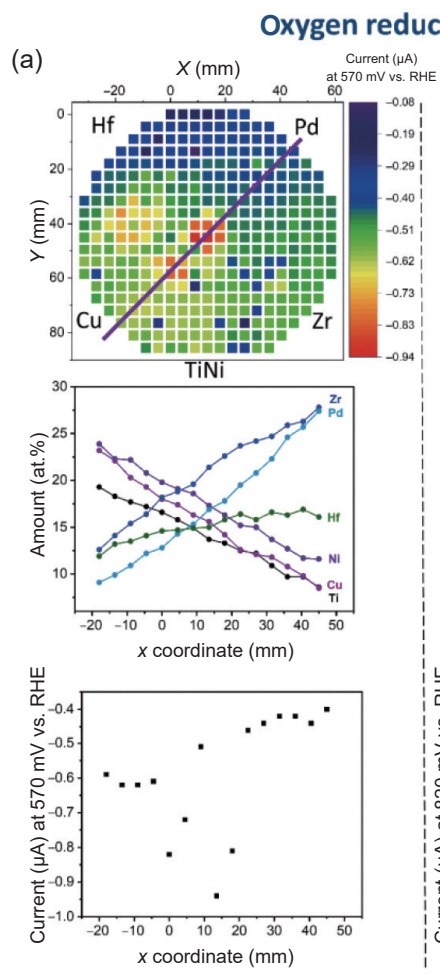
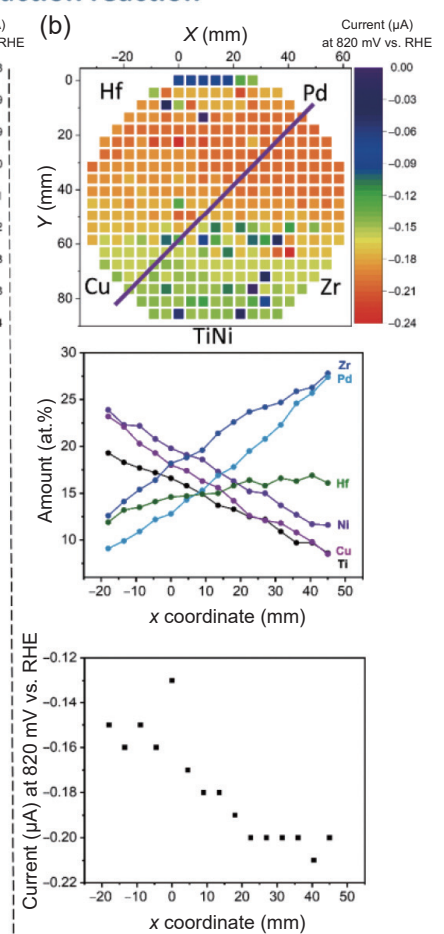

Hydrogen evolution reaction

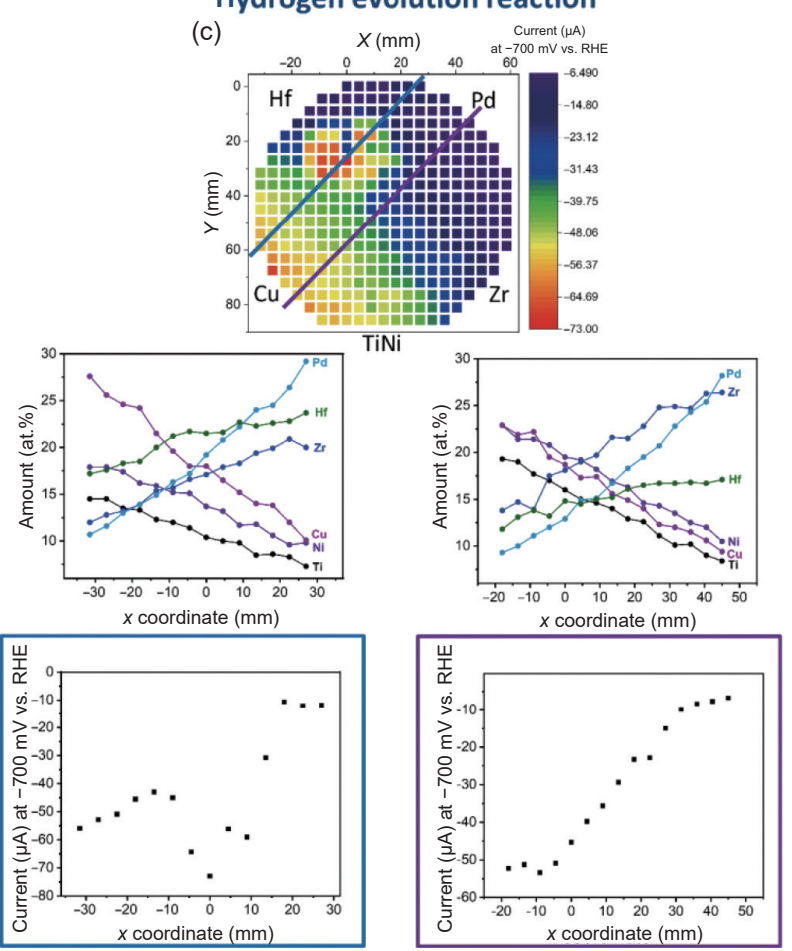

Figure 2 Correlation of the amount of each element (middle row) at each of the measurement areas marked with the lines on MLs (top row) and the measured current at $570 \mathrm{mV}$ and $820 \mathrm{vs}$. RHE for ORR and at $-700 \mathrm{mV}$ for HER (bottom row). Lines indicated in the activity maps (top row) determined the MAs used for this analysis, represented by their $x$ coordinate.

For HER, no matter the potential at which the activity map is measured (see Fig. S4 in the ESM), both the main trend with increasing activity from the upper right side (Fig. 2, from the high $\mathrm{Pd}$ side) of the ML to the lower left side (higher $\mathrm{Ti}, \mathrm{Ni}$ and $\mathrm{Cu}$ side) and the high activity area (at relatively high amounts of $\mathrm{Hf}$ and Pd, 20 at.\%-25 at.\%) remain the same. Interestingly, a clearly visible rise of the activity along this diagonal of the ML is opposite to the amount of Pd, which is known to be an efficient HER catalyst [27]. This observation indicates that the synergistic interactions of all elements incorporated in HEAs is more important for electrocatalytic activities than the content of particular elements and that in the case of the analysed ML, the active sites containing mostly $\mathrm{Cu}$ and $\mathrm{Ti} / \mathrm{Ni}$ are most efficient. In addition to the diagonal activity trend a narrow higher activity cluster is found located at the centre with a slight shift towards the area with higher Hf contents, which support the idea of possible synergetic interactions contributing to the activity of the catalyst, since Hf is not reported to be a highly active HER catalyst. The highest activity for HER is obtained for a composition of $\mathrm{Ti}_{11} \mathrm{Ni}_{13} \mathrm{Cu}_{18} \mathrm{Zr}_{17} \mathrm{Pd}_{19} \mathrm{Hf}_{22}$.

\section{Conclusion}

To conclude, we have presented a reliable and efficient, highthroughput method for screening a new high-entropy material system (TiNi)-Cu-Hf-Pd-Zr for electrocatalysis. To combine elements mostly used in heterogeneous catalysis as support ( $\mathrm{Ti}$, $\mathrm{Hf}, \mathrm{Zr}$ ) with metals commonly used in catalysis $(\mathrm{Cu}, \mathrm{Ni}, \mathrm{Pd})$ we selected such a composition and found previously unknown and surprising activity compositions for HER and ORR. These identified compositions definitively need the presence of also supposedly non-active elements. Interestingly, different elemental compositions regarding the relative amounts of the elements seem to boost different electrocatalytic reactions. For the ORR, the variation of $\mathrm{Ti}$ and $\mathrm{Ni}$ exhibits an important factor regarding the activity. Additionally, our measurements indicate that an intermediate amount of all elements in the material library reveals higher activity for the ORR. The HER in contradistinction occurs with higher activity at decreasing Pd contents, and in a small cluster of measurement areas in the middle of the material library. We would like to point out that the only feasible way to disentangle the complexity of the near infinite possible compositions with unknown physico-chemical interaction is to use screening of large numbers of material libraries to create large and growing data sets which at a later stage will serve as a basis for machine learning for improved predictions or starting compositions.

\section{Acknowledgements}

The authors acknowledge financial support by the German Research Foundation (Deutsche Forschungsgemeinschaft, DFG) in the framework of the projects AN 1570/2-1 (C. A., S. S.) and LU 1175/31-1) (A. L). This project has received funding from the European Research Council (ERC) under the European Union's Horizon 2020 research and innovation programme (grant agreement CasCat [833408], W. S.).

Funding note: Open Access funding enabled and organized by Projekt DEAL.

Electronic Supplementary Material: Supplementary material (EDS and XRD measurements, confirmation of reliability of electrochemical measurements) is available in the online version of this article at https://doi.org/10.1007/s12274-021-3637-z.

Open Access This article is licensed under a Creative Commons Attribution 4.0 International License, which permits use, sharing, adaptation, distribution and reproduction in any medium or format, as long as you give appropriate credit to the original author(s) and the source, provide a link to the Creative Commons licence, and indicate if changes were made. 
The images or other third party material in this article are included in the article's Creative Commons licence, unless indicated otherwise in a credit line to the material. If material is not included in the article's Creative Commons licence and your intended use is not permitted by statutory regulation or exceeds the permitted use, you will need to obtain permission directly from the copyright holder.

To view a copy of this licence, visit http://creativecommons. org/licenses/by/4.0/.

\section{References}

[1] Yeh, J. W.; Chen, Y. L.; Lin, S. J.; Chen, S. K. High-entropy alloys-A new era of exploitation. $M S F$ 2007, 560, 1-9.

[2] Guo, S.; Liu, C. T. Phase stability in high entropy alloys: Formation of solid-solution phase or amorphous phase. Progr. Nat. Sci. Mater. Int. 2011, 21, 433-446.

[3] Ye, Y. F.; Wang, Q.; Lu, J.; Liu, C. T.; Yang, Y. High-entropy alloy: Challenges and prospects. Mater. Today 2016, 19, 349-362.

[4] Löffler, T.; Savan, A.; Garzón-Manjón, A.; Meischein, M.; Scheu, C.; Ludwig, A.; Schuhmann, W. Toward a paradigm shift in electrocatalysis using complex solid solution nanoparticles. ACS Energy Lett. 2019, 4, 1206-1214.

[5] Wu, D. S.; Kusada, K.; Yamamoto, T.; Toriyama, T.; Matsumura, S.; Gueye, I.; Seo, O.; Kim, J.; Hiroi, S.; Sakata, O., et al. On the electronic structure and hydrogen evolution reaction activity of platinum group metal-based high-entropy-alloy nanoparticles. Chem. Sci. 2020, 11, 12731-12736.

[6] Gludovatz, B.; Hohenwarter, A.; Catoor, D.; Chang, E. H.; George, E. P.; Ritchie, R. O. A fracture-resistant high-entropy alloy for cryogenic applications. Science 2014, 345, 1153-1158.

[7] Ludwig, A. Discovery of new materials using combinatorial synthesis and high-throughput characterization of thin-film materials libraries combined with computational methods. npj Comput. Mater. 2019, 5, 70 .

[8] Ludwig, A.; Zarnetta, R.; Hamann, S.; Savan, A.; Thienhaus, S. Development of multifunctional thin films using high-throughput experimentation methods. Int. J. Mater. Res. 2008, 10, 1144-1149.

[9] Gao, S. J.; Hao, S. Y.; Huang, Z. N.; Yuan, Y. F.; Han, S.; Lei, L. C.; Zhang, X. W.; Shahbazian-Yassar, R.; Lu, J. Synthesis of highentropy alloy nanoparticles on supports by the fast moving bed pyrolysis. Nat. Commun. 2020, 11, 2016.

[10] Li, Z. M.; Ludwig, A.; Savan, A.; Springer, H.; Raabe, D. Combinatorial metallurgical synthesis and processing of highentropy alloys. J. Mater. Res. 2018, 33, 3156-3169.

[11] Glasscott, M. W.; Pendergast, A. D.; Goines, S.; Bishop, A. R.; Hoang, A. T.; Renault, C.; Dick, J. E. Electrosynthesis of highentropy metallic glass nanoparticles for designer, multi-functional electrocatalysis. Nat. Commun. 2019, 10, 2650.

[12] Qiu, H. J.; Fang, G.; Gao, J. J.; Wen, Y. R.; Lv, J.; Li, H. L.; Xie, G. Q.; Liu, X. J.; Sun, S. H. Noble metal-free nanoporous high-entropy alloys as highly efficient electrocatalysts for oxygen evolution reaction. ACS Materials Lett. 2019, 1, 526-533.

[13] Pedersen, J. K.; Batchelor, T. A. A.; Bagger, A.; Rossmeisl, J. HighEntropy alloys as catalysts for the $\mathrm{CO}_{2}$ and $\mathrm{CO}$ reduction reactions. ACS Catal. 2020, 10, 2169-2176.
[14] Chen, H.; Lin, W. W.; Zhang, Z. H.; Jie, K. C.; Mullins, D. R.; Sang, X. H.; Yang, S. Z.; Jafta, C. J.; Bridges, C. A.; Hu, X. B., et al. Mechanochemical synthesis of high entropy oxide materials under ambient conditions: Dispersion of catalysts via entropy maximization. ACS Materials Lett. 2019, 1, 83-88.

[15] Löffler, T.; Meyer, H.; Savan, A.; Wilde, P.; Garzón Manjón, A.; Chen, Y. T.; Ventosa, E.; Scheu, C.; Ludwig, A.; Schuhmann, W. Discovery of a multinary noble metal-free oxygen reduction catalyst. Adv. Energy Mater. 2018, 8, 1802269.

[16] Qiu, H. J.; Fang, G.; Wen, Y. R.; Liu, P.; Xie, G. Q.; Liu, X. J.; Sun, S. H. Nanoporous high-entropy alloys for highly stable and efficient catalysts. J. Mater. Chem. A 2019, 7, 6499-6506.

[17] Batchelor, T. A. A.; Löffler, T.; Xiao, B.; Krysiak, O. A.; Strotkötter, V.; Pedersen, J. K.; Clausen, C. M.; Savan, A.; Li, Y. J.; Schuhmann, W., et al. Complex solid solution electrocatalyst discovery by computational prediction and high-throughput experimentation. Angew. Chem., Int. Ed. 2021, 60, 6932-6937.

[18] Tsai, C. F.; Yeh, K. Y.; Wu, P. W.; Hsieh, Y. F.; Lin, P. Effect of platinum present in multi-element nanoparticles on methanol oxidation. J. Alloys Compd. 2009, 478, 868-871.

[19] Yusenko, K. V.; Riva, S.; Carvalho, P. A.; Yusenko, M. V.; Arnaboldi, S.; Sukhikh, A. S.; Hanfland, M.; Gromilov, S. A. First hexagonal close packed high-entropy alloy with outstanding stability under extreme conditions and electrocatalytic activity for methanol oxidation. Scr. Mater. 2017, 138, 22-27.

[20] Yao, Y. G.; Huang, Z. N.; Xie, P. F.; Lacey, S. D.; Jacob, R. J.; Xie, H.; Chen, F. J., Nie, A. M.; Pu, T. C.; Rehwoldt, M., et al. Carbothermal shock synthesis of high-entropy-alloy nanoparticles. Science 2018, 359, 1489-1494.

[21] Xie, P. F.; Yao, Y. G.; Huang, Z. N.; Liu, Z. Y.; Zhang, J. L.; Li, T. Y.; Wang, G. F.; Shahbazian-Yassar, R.; Hu, L. B.; Wang, C. Highly efficient decomposition of ammonia using high-entropy alloy catalysts. Nat. Commun. 2019, 10, 4011.

[22] Schäfer, D.; Mardare, C.; Savan, A.; Sanchez, M. D.; Mei, B.; Xia, W.; Muhler, M.; Ludwig, A.; Schuhmann, W. High-throughput characterization of Pt supported on thin film oxide material libraries applied in the oxygen reduction reaction. Anal. Chem. 2011, 1916-1923.

[23] Grote, J. P.; Zeradjanin, A. R.; Cherevko, S.; Savan, A.; Breitbach, B.; Ludwig, A.; Mayrhofer, K. J. J. Screening of material libraries for electrochemical $\mathrm{CO}_{2}$ reduction catalysts-Improving selectivity of $\mathrm{Cu}$ by mixing with Co. J. Catal. 2016, 343, 248-256.

[24] Sliozberg, K.; Schäfer, D.; Erichsen, T.; Meyer, R.; Khare, C.; Ludwig, A.; Schuhmann, W. High-throughput screening of thin-film semiconductor material libraries I: System development and case study for Ti-W-O. ChemSusChem 2015, 8, 1270-1278.

[25] Batchelor, T. A. A.; Pedersen, J. K.; Winther, S. H.; Castelli, I. E.; Jacobsen, K. W.; Rossmeisl, J. High-entropy alloys as a discovery platform for electrocatalysis. Joule 2019, 3, 834-845.

[26] Löffler, T.; Savan, A.; Meyer, H.; Meischein, M.; Strotkötter, V.; Ludwig, A.; Schuhmann, W. Design of complex solid-solution electrocatalysts by correlating configuration, adsorption energy distribution patterns, and activity curves. Angew. Chem., Int. Ed. 2020, 59, 5844-5850.

[27] Sarkar, S.; Peter, S. C. An overview on Pd-based electrocatalysts for the hydrogen evolution reaction. Inorg. Chem. Front. 2018, 5, 2060-2080. 